\title{
Potential antimicrobial agents for the treatment of multidrug-resistant tuberculosis
}

\author{
Noor Alsaad ${ }^{1}$, Bob Wilffert',2, Richard van Altena ${ }^{3}$, Wiel C.M. de Lange ${ }^{3}$, \\ Tjip S. van der Werf ${ }^{4}$, Jos G.W. Kosterink ${ }^{1,2}$ and Jan-Willem C. Alffenaar ${ }^{1}$
}

Affiliations: 'University of Groningen, University Medical Center Groningen, Dept of Hospital and Clinical Pharmacy, Groningen, ${ }^{2}$ University of Groningen, Section of Pharmacotherapy and Pharmaceutical Care, Dept of Pharmacy, Groningen, ${ }^{3}$ University Medical Center Groningen, Tuberculosis Center Beatrixoord, Haren, and ${ }^{4}$ University of Groningen, University Medical Center Groningen, Depts of Internal Medicine and Pulmonary Diseases and Tuberculosis, Groningen, The Netherlands.

Correspondence: J-W.C. Alffenaar, Dept of Hospital and Clinical Pharmacy, University of Groningen, University Medical Center Groningen, P.O. Box 30.001, 9700 RB Groningen, The Netherlands.

E-mail: j.w.c.alffenaaraumcg.nl

ABSTRACT Treatment of multidrug-resistant (MDR) tuberculosis (TB) is challenging because of the high toxicity of second-line drugs and the longer treatment duration than for drug-susceptible TB patients. In order to speed up novel treatment for MDR-TB, we suggest considering expanding the indications of already available drugs. Six drugs with antimicrobial activity (phenothiazine, metronidazole, doxycycline, disulfiram, tigecycline and co-trimoxazole) are not listed in the World Health Organization guidelines on MDR-TB treatment but could be potential candidates for evaluation against Mycobacterium tuberculosis.

A systematic review was conducted to evaluate antituberculous activity of these drugs against M. tuberculosis. We searched PubMed, Google Scholar and Embase for English articles published up to December 31, 2012.

We reviewed in vitro, in vivo and clinical antituberculous activity of these drugs in addition to pharmacokinetics and side-effects. Of the drugs effective against actively replicating $M$. tuberculosis, cotrimoxazole seems to be the most promising, because of its consistent pharmacokinetic profile, easy penetration into tissue and safety profile. For the dormant state of $\mathrm{TB}$, thioridazine may play a potential role as an adjuvant for treatment of MDR-TB. A strategy consisting of pharmacokinetic/pharmacodynamic studies, dose finding and phase III studies is needed to explore the role of these drugs in MDR-TB treatment.

@ERSpublications

Six antimicrobial drugs are not listed in WHO guidelines on MDR-TB treatment but could offer potential for TB treatment http://ow.ly/q0HRV

Received: July 042013 | Accepted after revision: Aug 162013 | First published online: Aug 292013

Conflict of interest: Disclosures can be found alongside the online version of this article at www.erj.ersjournals.com 


\section{Introduction}

Multidrug-resistant (MDR) tuberculosis (TB) is defined as an infectious disease caused by Mycobacterium tuberculosis that is resistant to at least the two most powerful drugs currently known: isoniazid and rifampicin [1-4]. Treatment of MDR-TB is complex, using toxic drugs that must be administered for a longer duration than those used for drug-susceptible TB patients, and with lower likelihood of treatment success [5].

The loss of rifampicin in the treatment regimen of MDR-TB is particularly important, because although many drugs have the potential to kill rapidly dividing, metabolically active mycobacteria, few drugs are active when the population of $M$. tuberculosis has switched its genetic programme to a quiescent, dormant phenotype. These persisters provide the major challenge for the immune system as well as for drug treatment to achieve sterility [6].

The World Health Organization (WHO) estimates that 2.5\% of all TB patients and 3\% of all new cases are infected with MDR-TB [7, 8]; an estimated 650000 prevalent cases of MDR-TB occurred globally in 2010 [9]. The proportions of new TB cases with MDR-TB in Eastern European countries are at alarming levels, ranging from $19.4 \%$ to $32.3 \%$ [10]. One recent report from Belarus even indicates that almost half of newly diagnosed treatment-naïve patients with TB actually have MDR-TB. Furthermore, the fact that patients aged $<35$ years showed twofold higher odds of MDR-TB than those aged $\geqslant 35$ years suggests that emergence of MDR-TB is rampant in Belarus [11].

Most of the drug resistance is caused by mutations in genes of $M$. tuberculosis coding for drug targets, but there is also evidence for mutations resulting in upregulation of bacterial efflux pumps, potentially reducing susceptibility to several drug groups [12,13]. An important feature of M. tuberculosis is that under stress conditions, including hypoxia and host factors like nitric oxide, the organism is able to change its genetic programme by, for example, inducing a 48-gene regulon via the response regulator DosR; this leads to inhibited aerobic respiration, thereby suppressing M. tuberculosis replication [14].

It is clear that new antituberculous drugs are urgently needed. Unfortunately, development of new drugs is time-consuming, difficult and expensive, and low- and middle-income countries that comprise 95\% of TB cases around the world have limited resources for health expenditure as well as lack of support for research [15]. In order to speed up novel treatments for MDR-TB, in this review, we explore the possibility of expanding the indications of drugs already on the market.

We performed a systematic review of the published literature to select and describe antimicrobial drugs that are not listed in WHO guidelines on MDR-TB but may have in vitro and/or in vivo or clinical antituberculous activity, and are already available on the market labelled for other diseases. Based on these data, in addition to pharmacokinetic parameters, administration route, safety and tolerance of the selected drugs, suggestions are made as to which drug is promising so that it can be further evaluated for MDR-TB treatment.

\section{Search strategy and selection criteria}

To select antimicrobial drugs for our systematic review that have activity against $M$. tuberculosis, we used the following search strategy in PubMed: "antimicrobial drugs AND tuberculosis AND 2012". To assess the activity of the selected drugs, we performed a second search in the databases PubMed, Google Scholar and Embase for published, original and relevant literature with search terms that included but were not restricted to the name of each drug combined with $M$. tuberculosis. Selection criteria were activity of the selected drug against $M$. tuberculosis in vitro, in vivo and/or in clinical studies. In vitro studies that described susceptibility to the drug of interest were selected. In vivo also included animal studies infected with M. tuberculosis describing the logarithmic decline in colony-forming units (CFU) of M. tuberculosis per kilogram of body mass following drug administration. Clinical studies included clinical trials that described efficacy, sputum culture conversion and clinical outcome in MDR-TB patients. Further cross-references were obtained manually from bibliographies of the identified and relevant papers and books. Englishlanguage publications covering all dates from the creation of each database up to December 31, 2012 were included. To evaluate relevant pharmacokinetic parameters, safety and tolerance of the drugs, studies in TB patients were selected. If the latter were not available, representative data for the drugs were presented. The results were divided per drug, using the subheadings in vitro, in vivo and clinical data within each case. A flow chart is presented in figure 1.

\section{Results}

Based on the first search strategy, 783 publications were retrieved. Screening of the titles and abstracts of these publications resulted in selection of six antimicrobial drugs that are on the market and are not listed 

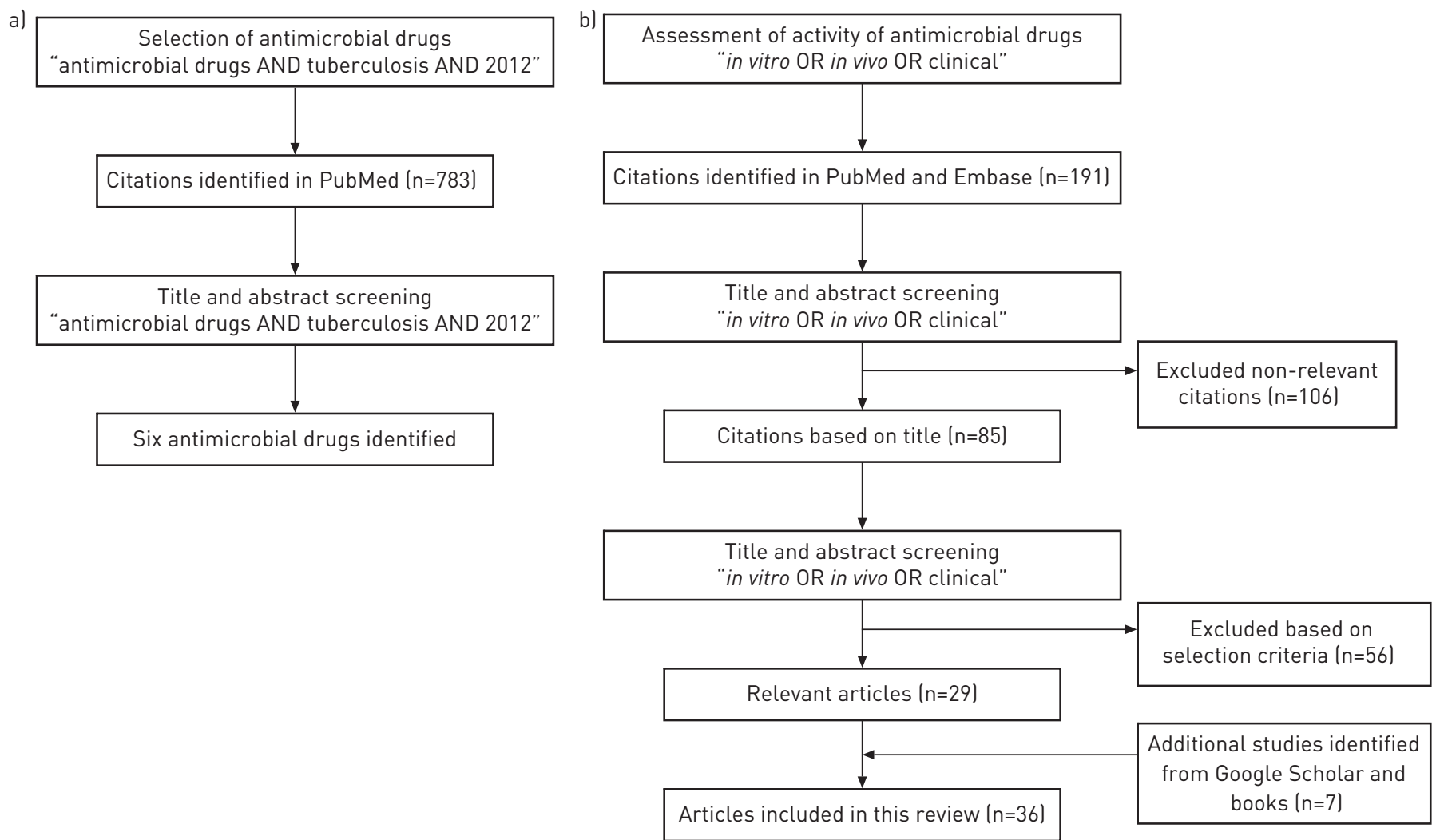

FIGURE 1 Flow chart of the selection process for retrieved publications. a) Selection of antimicrobial drugs and b) selection of publications.

for $\mathrm{TB}$ in WHO guidelines. The selected antimicrobial drugs were phenothiazines (thioridazine and chlorpromazine), metronidazole, tetracyclines, disulfiram and co-trimoxazole (sulfamethoxazole-trimethoprim) (SXT).

Our second search strategy resulted in 191 published articles. Based on screening of the titles, 85 articles were selected and 106 articles were excluded because of irrelevant titles. The abstracts of 85 articles were screened; 56 were excluded because the selection criteria were not met. Finally, 29 relevant articles in addition to four articles from Google Scholar and three references from books were included in the systematic review. The search in Embase did not result in any additional articles compared to PubMed. The results for each drug are discussed in detail below. A summary of the results, based on in vitro, in vivo and clinical data, is provided in table 1. Mechanisms of actions of these drugs are described in figure 2. The pros and cons of the selected drugs are summarised in table 2. The clinically evaluated dose and the effect of the drugs against $\mathrm{TB}$ are summarised in table 3.

\section{Phenothiazines: thiorizadine}

Phenothiazines such as thioridazine, chlorpromazine and promethazine belong to the group of antipsychotic drugs [59]. The phenothiazine derivative thioridazine is a neuroleptic compound that has been used for over four decades [60]. Phenothiazines have the ability to inhibit the bacterial efflux pump that protects the bacterial cell against harmful substances [59]. Thioridazine not only inhibits specific efflux pump systems but also the expression of genes that code for efflux pumps of M. tuberculosis [61].

\section{In vitro studies}

Phenothiazine family compounds, including chlorpromazine and thioridazine, are known to possess appreciable levels of antimicrobial activity against $M$. tuberculosis organisms in vitro. The anti-MDR-TB activity of thioridazine and chlorpromazine was similar [43]. These compounds may be concentrated more than tenfold by macrophages that phagocytise M. tuberculosis [44]. It is likely that the concentration of either phenothiazine required to kill $\mathrm{M}$. tuberculosis cells in vitro is achieved because the macrophage is not only able to concentrate phenothiazines but also to make these compounds available in an active form to the cytoplasmic structure of the macrophage that houses the entrapped phagocytised bacterium [43]. Thioridazine inhibits the growth of clinical isolates of M. tuberculosis that are resistant to streptomycin, 


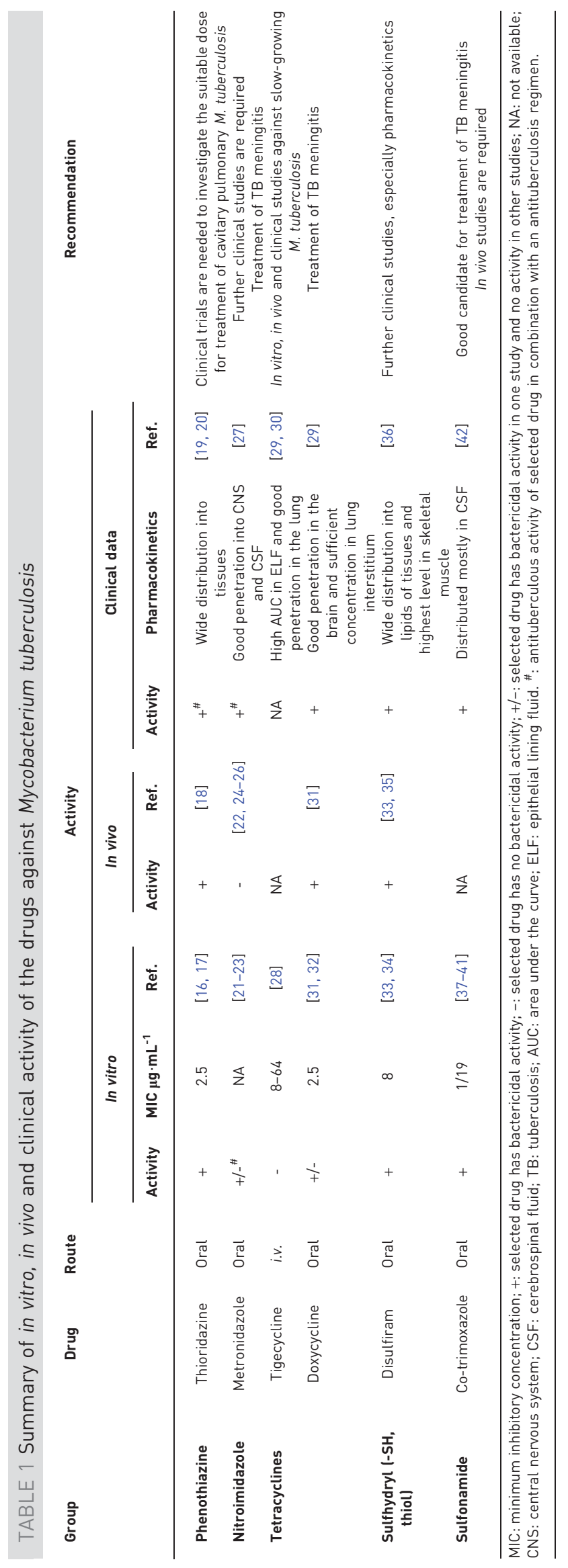




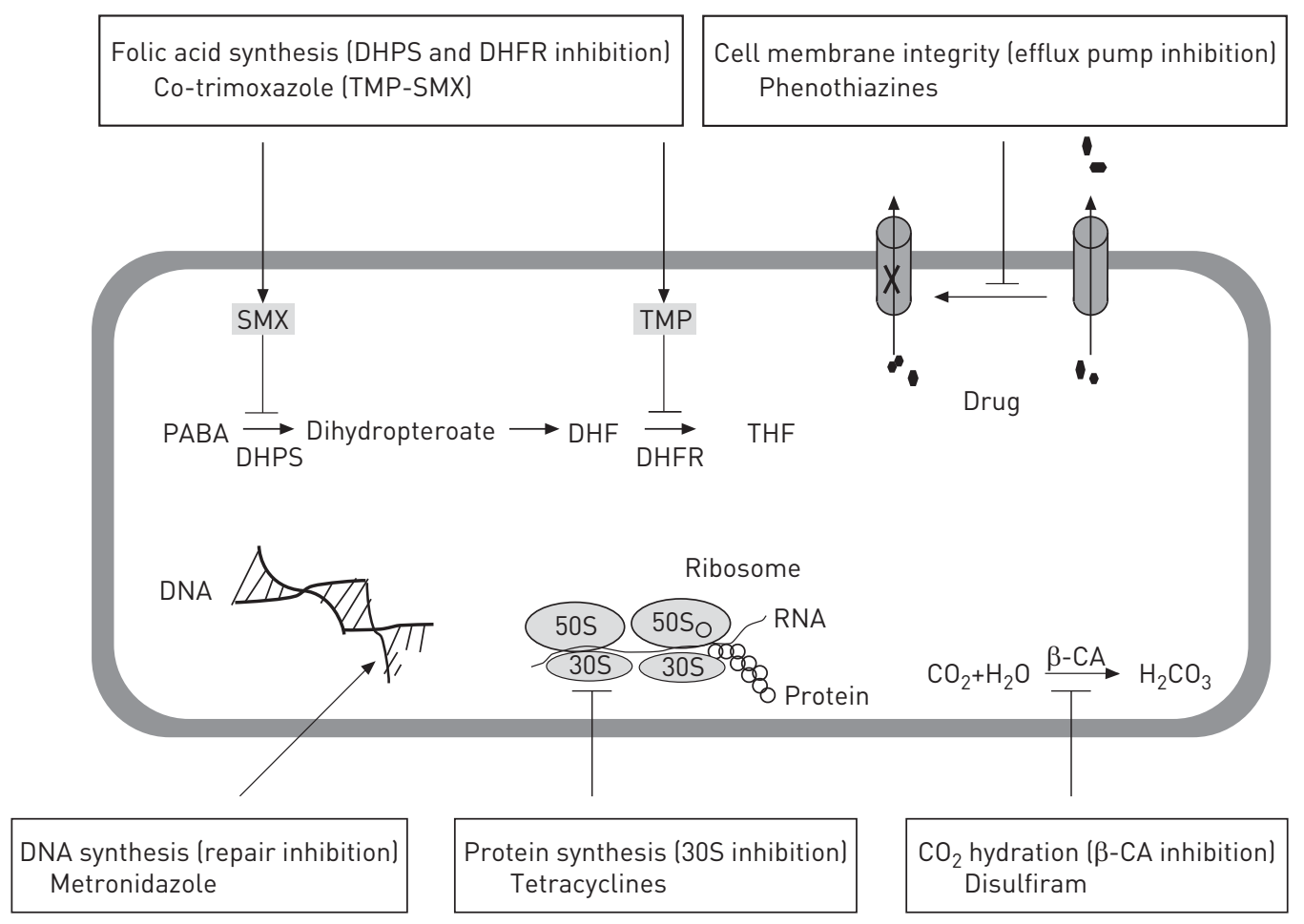

FIGURE 2 The mechanisms of action of the selected drugs. PABA: p-aminobenzoic acid; DHPS: dihydropteroate synthase; DHF: dihydrofolate; DHFR: DHF reductase; THF: tetrahydrofolic acid; SMX: sulfamethoxazole; TMP: trimethoprim; $\beta$-CA: $\beta$-class carbonic anhydrase.

rifampicin, isoniazid, ethambutol and pyrazinamide (first-line antituberculous drugs) [16]. The minimum inhibitory concentration of thioridazine required for $50 \%$ inhibition of M. tuberculosis $\mathrm{H} 37 \mathrm{Rv}$ clinical isolates is $2.5 \mu \mathrm{g} \cdot \mathrm{mL}^{-1}$ [17]. Because thioridazine does not appear toxic to the macrophage in vitro, it could be used in the treatment of intracellular M. tuberculosis infections [43].

\section{In vivo studies}

Groups of five female BALB/c mice were injected intraperitoneally with $1 \times 10^{6} \mathrm{CFU} \cdot \mathrm{mL}^{-1}$ of $M$. tuberculosis and treated with thioridazine in a dose range of $0.05-0.5 \mathrm{mg} \cdot \mathrm{day}^{-1}$, which is equivalent to that used for psychosis in humans $\left(1200 \mathrm{mg} \cdot \mathrm{day}^{-1}\right)$. There was a $>5$-log reduction in the number of CFU per millilitre derived from the lungs of infected mice compared with the control group within 1 month [18].

\section{Clinical studies}

When thioridazine is administered orally it is rapidly absorbed, with peak plasma concentrations occurring within 2-3 h. Thioridazine is widely distributed in tissues like the liver, blood and kidney [19, 20], yet it is distributed less favourably to the brain [45]. It is not useful for treatment of cavitary pulmonary M. tuberculosis because the concentration of thioridazine required for killing or inhibiting M. tuberculosis outside the macrophage exceeded the concentration that could be achieved in patients receiving standard dosages [43].

Thioridazine undergoes 5-sulfoxidation and N-demethylation by cytochrome P450 (CYP)1A2 and CYP3A4, while CYP2D6 catalyses mono- and di-2-sulfoxidation of thioridazine in the human liver. CYP2D6 and CYP3A4 catalyse thioridazine mono-2-sulfoxidation [62]. Thioridazine is almost entirely $(99.85 \%)$ bound to serum proteins; its metabolites are bound to serum proteins to a lesser extent. This could be a problem because a small change in the binding capacity of proteins can alter the unbound concentration of thioridazine that has clinical activity. The unbound concentrations of thioridazine metabolites that include thioridazine side chain sulfoxide and thioridazine side chain sulfone are higher (two and nine times, respectively) than the unbound concentration of thioridazine [46].

Thioridazine can be used as adjuvant for regimens already containing several other drugs for treatment of MDR-TB. It is administered in drug-resistant TB until the susceptibility of strains is known [44]. 
TABLE 2 Pros and cons of the six selected antimicrobial drugs

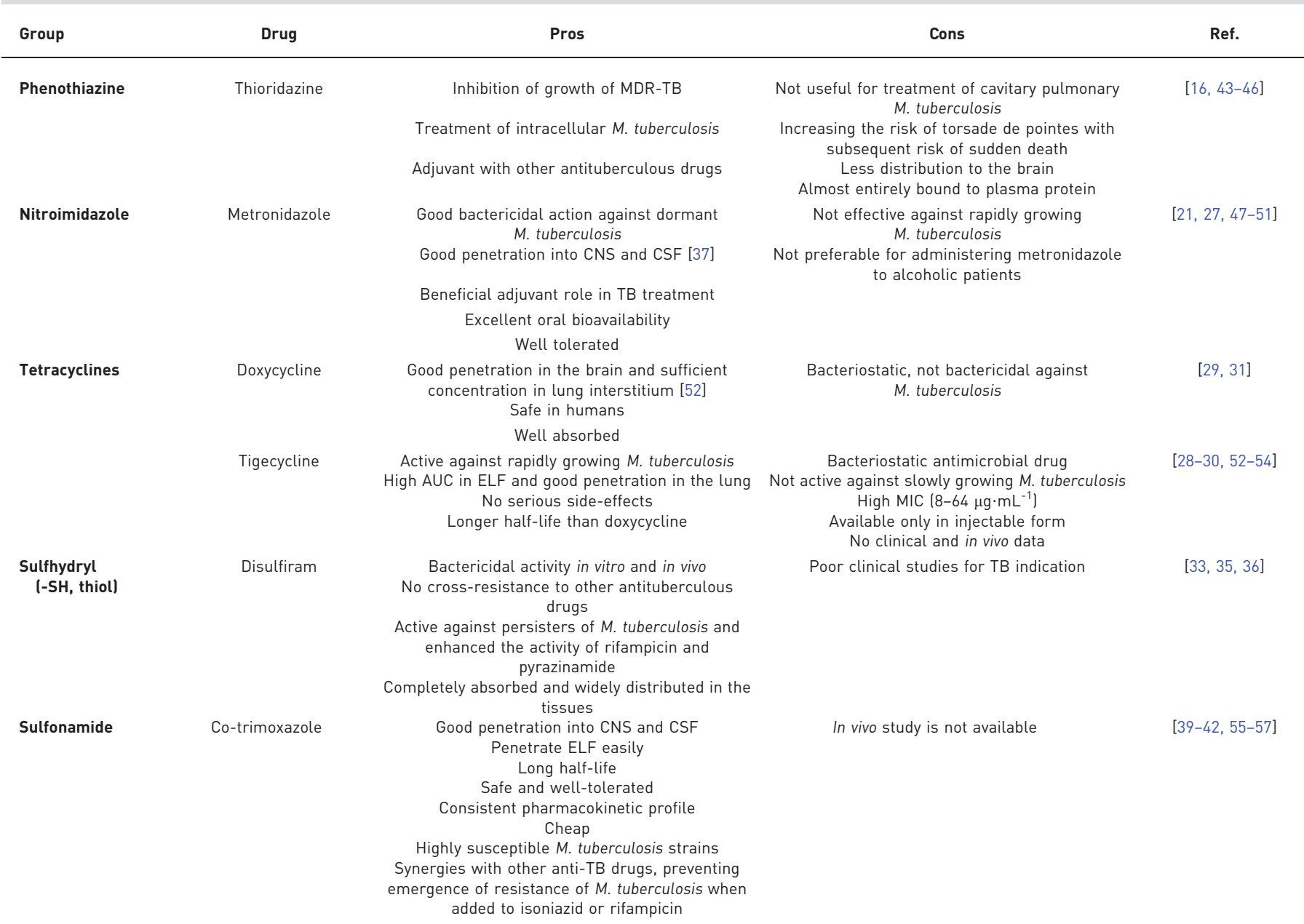

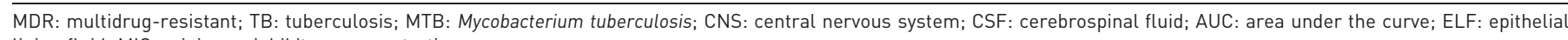
lining fluid; MIC: minimum inhibitory concentration.

Of 17 extensively drug-resistant (XDR)-TB patients, 14 were treated with thioridazine in combination with linezolid and/or moxifloxacin at a daily dose of only $25 \mathrm{mg}$ for 2 weeks, after which the dose was increased by $25 \mathrm{mg}$ weekly until it reached $200 \mathrm{mg} \cdot \mathrm{day}^{-1}$. The combined therapy of thioridazine, moxifloxacin and linezolid cured $61 \%$ of patients, and $22 \%$ of patients who were still on follow-up showed a beneficial response. Thioridazine was discontinued in two patients because of pancytopenia in one and allergic dermatitis in the other. In this study, the authors speculate that thioridazine could have contributed to an earlier bacteriological sputum conversion. No prolongation of the QT interval or other cardiac complication was observed in those patients who received thioridazine. Combined therapy including linezolid, moxifloxacin and thioridazine was associated with a relapse-free cure in most cases [58]. The quality of life of the patients was improved shortly after the inclusion of thioridazine in their regimens; most of them benefited from less night sweating, increased appetite and weight gain, and decreased levels of anxiety [58]. In another study, thioridazine cured 10 out of 12 patients; the other two patients also responded, but they dropped out of the programme [63].

The side-effects of thioridazine, as for all phenothiazines, are dose-dependent and include QTc prolongation, thereby increasing the risk of torsade de pointes with subsequent risk of sudden death [64]. To avoid the risk of sudden death in patients whose QTc interval is significantly increased, patients should be screened by ECG before and during treatment with thioridazine [63]. Because chlorpromazine causes frequent and serious side-effects when administered chronically, it is not a good candidate drug for treatment of MDR-TB [65]. 


\section{TABLE 3 Clinical studies showing response of tuberculosis (TB) patients to the selected drugs}

\begin{tabular}{|c|c|c|c|c|c|}
\hline Drug & Treatment regimen & Patient population & Dose regimen & Response & Ref. \\
\hline Thioridazine & $\begin{array}{l}\text { Combination with linezolid } \\
\text { and/or moxifloxacin }\end{array}$ & XDR-TB patients & $\begin{array}{l}25 \mathrm{mg} \text { per day for } 2 \text { weeks; } \\
\text { followed by a dose increase of } \\
25 \mathrm{mg} \text { weekly until } \\
\text { the maximum dose of } 200 \mathrm{mg} \\
\text { per day was reached }\end{array}$ & $\begin{array}{l}\text { Earlier bacteriological sputum conversion } \\
\text { Relapse-free cure }\end{array}$ & {$[58]$} \\
\hline Metronidazole & $\begin{array}{l}\text { Antituberculous regimen } \\
\text { (streptomycin, isoniazid and } \\
\text { rifampicin) }\end{array}$ & $\begin{array}{l}\text { Advanced pulmonary } \\
\text { TB patients }\end{array}$ & $\begin{array}{l}400 \mathrm{mg} \text { three times per day for } \\
\qquad 8 \text { weeks }\end{array}$ & $\begin{array}{l}\text { Significant improvement in clinical response } \\
\text { Reduction of sputum quantity } \\
\text { Enhanced radiographic improvement } \\
\text { Improvement in susceptibility to anti-TB drugs }\end{array}$ & {$[50]$} \\
\hline Doxycycline & & TB patients & $20 \mathrm{mg}$ three times per day & $\begin{array}{c}\text { Suppress immunopathologic MMPs to reduce } \\
\text { tissue damage }\end{array}$ & [31] \\
\hline Tigecycline & & & & No clinical data available & \\
\hline \multicolumn{6}{|l|}{ Disulfiram } \\
\hline Co-trimoxazole & $\begin{array}{l}\text { With antituberculous } \\
\text { regimen }\end{array}$ & MDR-TB patients & 480 mg, 960 mg per day & $\begin{array}{l}\text { No treatment discontinuation } \\
\text { No serious side-effects }\end{array}$ & [55] \\
\hline
\end{tabular}

XDR: extensively drug-resistant; MMPs: matrix metalloproteinases; MDR: multidrug-resistant.

\section{Metronidazole}

Metronidazole is currently licensed for the oral treatment of infections by protozoa (trichomoniasis and amoebiasis) and anaerobic bacteria [47]. This drug has only been evaluated to a limited extent for TB [47], showing good bactericidal action in anaerobic conditions against dormant TB bacilli, but has no effect on aerobic cultures of $M$. tuberculosis [21, 47, 48].

Bactericidal activity of metronidazole depends on the formation of a redox intermediate metabolite from the reduction of the nitro group in metronidazole under anaerobic conditions. This metabolite oxidises DNA and causes extensive breakage of DNA strands and subsequent cell death, and also inhibits DNase 1, which has a function as a repair endonuclease in bacteria. Hence, reduced metronidazole exerts a dual action by breaking DNA strands and inhibiting the enzyme responsible for repairing strand breaks in DNA $[21,27,66]$.

\section{In vitro studies}

Metronidazole showed no effect when added to bone marrow-derived macrophages infected with M. tuberculosis and did not decrease the bacterial load, although a high concentration of metronidazole was used [22]. Adding metronidazole to a regimen of rifampicin, moxifloxacin and amikacin and/or capreomycin significantly improved killing of dormant (anaerobic and drug-tolerant) M. tuberculosis in an adipocyte model [23].

An in vitro study was conducted to determine the effect of metronidazole against $M$. tuberculosis incubated under aerobic conditions. Although a high concentration of metronidazole was used $\left(512 \mu \mathrm{g} \cdot \mathrm{mL}^{-1}\right)$, it had no effect on aerobic cultures of $M$. tuberculosis. However, under anaerobic conditions, when $32 \mu \mathrm{g} \cdot \mathrm{mL}^{-1}$ of metronidazole was incubated with $M$. tuberculosis alone and in combination with either rifampicin or isoniazid, this caused a reduction of $69 \%$ and $95 \%$ in $\mathrm{CFU} \cdot \mathrm{mL}^{-1}$, respectively. Again in this study, 8 days of anaerobic exposure of dormant bacilli to $8 \mu \mathrm{g} \cdot \mathrm{mL}^{-1}$ of metronidazole caused a 2.7 -log reduction in the number of CFU. Rifampicin enhanced the bactericidal activity of metronidazole when used in combination, leading to a 3.68-log unit decline in $\mathrm{CFU} \cdot \mathrm{mL}^{-1}[21]$.

\section{In vivo studies}

Although metronidazole had no effect on the growth of $M$. tuberculosis in lungs of aerosol-infected mice at a dose of $15 \mathrm{mg} \cdot \mathrm{kg}^{-1}$, there was a relatively small but statistically significant reduction in bacterial counts during a chronic phase of disease. This chronic phase, in which no marked changes in bacterial load can usually be detected, established after the onset of acquired cellular immunity in mice as a result of the progressively growing $M$. tuberculosis, resulted in a proportion of bacilli shifting into a dormant state $[22,67]$. The possible explanation for the low activity of metronidazole is that $M$. tuberculosis is not in a state of anaerobic metabolism in which it is susceptible to metronidazole [22].

In another in vivo study using a granuloma model of $M$. tuberculosis dormancy (mouse hollow-fibre model), metronidazole $\left(100 \mathrm{mg} \cdot \mathrm{kg}^{-1}\right)$ failed to show a beneficial effect against bacilli, although there was immunohistochemical and mutant survival-based evidence of tissue hypoxia. The possible explanations for this result could be a less-than-optimal concentration of metronidazole, poor penetration into granulomatous lesions or lack of hypoxic conditions to permit reductive activation of metronidazole [24]. 
Metronidazole at 50 and $100 \mathrm{mg} \cdot \mathrm{kg}^{-1}$ in guinea pigs infected with $M$. tuberculosis combined with standard regimens showed no significant activity, perhaps because of poor penetration of metronidazole into the necrotic core of the hypoxic granulomas or the concentrations of metronidazole effective against M. tuberculosis under completely anaerobic and microaerophilic conditions [25]. These results were comparable to the in vivo study, where treatment of $\mathrm{BALB} / \mathrm{c}$ and $\mathrm{C} 3 \mathrm{HeB} / \mathrm{FeJ}$ mice that developed highly organized necrotic lesions following a TB infection, called the Kramnik model, with $200 \mathrm{mg} \cdot \mathrm{kg}^{-1}$ metronidazole for 7-8 weeks had no bactericidal activity against M. tuberculosis, although necrotic lesions in the Kramnik model showed evidence of hypoxia [26].

\section{Clinical studies}

The oral bioavailability of metronidazole is almost complete (98.9\%) [49]. Peak serum levels are reached within 1-3 h [68]. 10-20\% metronidazole is bound to plasma proteins [68]. The half-life of metronidazole is about $8 \mathrm{~h}$ [49]. Metronidazole is distributed in different tissues with various percentages of penetration, and it has good penetration into the cerebrospinal fluid (CSF) and central nervous system (CNS) [27]. It is metabolised in the liver, resulting in the formation of two oxidation products: the alcohol metabolite 1-(2-hydroxyethyl)-2-hydroxymethyl-5-nitroimidazole, which has an antimicrobial activity of 30-75\% compared with metronidazole; and the acidic metabolite 2-methyl-5-nitroimidazole-1-acetic acid, which has $5 \%$ of the activity metronidazole and is only detected in patients with renal dysfunction [69, 70]. Metronidazole and its metabolites are mainly excreted in urine [71].

There are few clinical studies of metronidazole for TB. In a single-blinded study, metronidazole (400 mg three times daily) or a placebo were administered for the first 8 weeks in addition to the standard antituberculous regimen. Addition of metronidazole resulted in a significant improvement in clinical response, with reduction of sputum quantity, enhanced radiographic improvement and improved sensitivity to antituberculousdrugs compared with the placebo group. The results showed a $56 \%$ improvement in the sensitivity of TB bacilli to antituberculous drugs on follow-up over 12 weeks in the metronidazole group, compared with $38 \%$ in placebo group. This study confirmed that metronidazole has a beneficial adjuvant role in the treatment of TB [50].

In an ongoing clinical trial, metronidazole is being used in MDR-TB patients at a dose of $500 \mathrm{mg}$ three times daily in combination with standard second-line antituberculous drugs. The results of this study are expected soon [72].

Metronidazole is generally well-tolerated. Adverse reactions include reversible neutropenia, minor gastrointestinal side-effects, metallic taste, vaginal and urethral burning, and darkening of the urine. CNS side-effects include ataxia, vertigo, peripheral neuropathy and headache [73]. Metronidazole can produce a reaction similar to that of disulfiram when administered to patients using alcohol because the interaction between metronidazole and ethanol leads to a toxic accumulation of acetaldehyde in the blood [51]. Care should be taken in using this drug in alcohol-abuse patients.

\section{Tetracyclines}

Doxycycline and tigecycline belong to the tetracycline group of antimicrobials, which exhibit a broad spectrum of activity against different pathogens, including Gram-positive and Gram-negative bacteria [29]. Tetracyclines are bacteriostatic and act by binding to the bacterial $30 \mathrm{~S}$ ribosomal subunit and inhibiting protein synthesis. Tetracyclines are effective in the treatment of a wide range of infectious diseases including community-acquired respiratory tract infection, sexually transmitted diseases and skin conditions [29].

Doxycycline is an antibiotic with broad spectrum matrix metalloproteinase (MMP) inhibitory activity [74]. It not only reduces the expression of MMPs, thereby minimising tissue damage in TB, but also suppresses mycobacterial growth. Because doxycycline is safe, cheap and almost universally available, it may represent a new adjunctive therapy to improve outcomes and reduce mortality for TB [31]. Tigecycline (GAR-936) is a new semisynthetic glycylcycline (tetracycline analogue) and is effective against intra-abdominal, skin and soft-tissue infections caused by staphylococci, enterococci or streptococci, as well as most Enterobacteriaceae and anaerobic pathogens [29, 52]. Tigecycline is effective against rapid-growing mycobacteria (Mycobacterium fortuitum, Mycobacterium chelonae and Mycobacterium abscessus) but showed no activity against more slow-growing mycobacteria (M. tuberculosis) [52, 53].

\section{In vitro studies}

Doxycycline suppressed MMP1 and MMP3 as well as tumour necrosis factor- $\alpha$ secretion from primary human macrophages infected with $M$. tuberculosis at $72 \mathrm{~h}$ in a dose-dependent manner. This drug is bacteriostatic to $M$. tuberculosis clinical isolates with an minimum inhibitory concentration (MIC) of $2.5 \mu \mathrm{g} \cdot \mathrm{mL}^{-1}$ [31]. In $69 \mathrm{MDR}-\mathrm{TB}$ isolates obtained from patients in the Samara region of Russia, five (7.4\%) 
isolates were resistant to doxycycline [32]. In vitro MIC values of tigecycline against clinical isolates of M. tuberculosis were as high as $8-64 \mu \mathrm{g} \cdot \mathrm{mL}^{-1}[28]$.

\section{In vivo studies}

Doxycycline decreased mycobacterial replication in infected guinea pigs but showed no effect on MMP activity. At a dose of 5 or $20 \mathrm{mg} \cdot \mathrm{kg}^{-1}$, doxycycline monotherapy suppressed lung CFU at 10 weeks in a dose-dependent manner. The results showed that doxycycline improved the outcome for TB by acting directly on mycobacterial proliferation rather than on MMP activity [31]. No publications mentioned the antimicrobial activity of tigecycline in vivo.

\section{Clinical studies}

Doxycycline is available in oral form, unlike tigecycline, which is only available in an injectable form [29]. This drug is absorbed well from the gastrointestinal tract, with bioavailability ranging between $75 \%$ and $100 \%$ [29]. It is absorbed quickly and reaches its maximum serum concentration within $4 \mathrm{~h} \mathrm{[29].}$ Maximum serum concentration and area under the plasma concentration time curve (AUC) of tigecycline are proportional to the dose $[29,52]$. In epithelial lining fluid, the AUC of tigecycline was $2.28 \mu \mathrm{g} \cdot \mathrm{h}^{-1} \cdot \mathrm{mL}^{-1}$, which is higher than that of serum $\left(1.73 \pm 0.64 \mu \mathrm{g} \cdot \mathrm{h}^{-1} \cdot \mathrm{mL}^{-1}\right)$ [30]. Doxycycline is lipophilic and penetrates well into tissues, especially the brain, eye, prostate and intestinal epithelia [29]. Higher concentrations of doxycycline are also found in the kidney and liver [75]. Tigecycline has a large volume of distribution, 7-10 L $\cdot \mathrm{kg}^{-1}$ [76]. It shows good penetration into tissues like bones, skin, liver and lung [29]. The protein binding of doxycycline is 60-95\%, while that of tigecycline ranges between $71 \%$ and $89 \%$ [29].

Tigecycline has a half-life of $15-36 \mathrm{~h}$, which is greater than the 12-h half-life of doxycycline [29, 30]. No more than $15 \%$ of tigecycline is excreted in the urine in an unchanged form $[29,54]$. About $30-40 \%$ of doxycycline is excreted unchanged in the urine [29].

Doxycycline is safe in humans and may suppress immunopathological MMPs, thereby reducing tissue damage in TB patients using a low dose (20 mg twice daily). It may achieve sufficient concentration in the lung interstitium to decrease the growth of M. tuberculosis and modulate MMP activity and expression [31]. No clinical data are available for the potential use of tigecycline for the treatment of MDR-TB in humans. The most common side-effects of doxycycline are gastrointestinal problems and skin reactions [77]. In a study with healthy subjects, tigecycline had no serious side-effects except for nausea and vomiting, which were dose-related [54].

\section{Disulfiram}

Disulfiram (tetraethylthiuram disulfide, DSF) has been used orally in the clinical treatment of alcoholism since 1949. DSF is a prodrug and is enzymatically metabolised in the blood to metabolites, primarily diethyldithiocarbamate (DDC or DETC) within 4 min [33, 35]. DSF and DDC exhibit growth-inhibitory activity against bacteria, fungi, protozoa and viruses. DSF is effective against MDR/XDR-TB and exhibits bactericidal activity in vivo and in vitro [35]. The mechanism of action of DDC against M. tuberculosis has been reported as inhibition of $\beta$-class carbonic anhydrases from M. tuberculosis [78].

\section{In vitro studies}

Peripheral blood mononuclear cells of healthy and HIV subjects were preincubated with $100-1000 \mathrm{ng} \cdot \mathrm{mL}^{-1}$ of DDC and then infected with M. tuberculosis H37Rv. DDC reduced the CFU number of these intracellular mycobacteria. DDC also enhances the antimycobacterial activity of monocyte-derived macrophages from healthy volunteers injected with $5 \mathrm{mg} \cdot \mathrm{kg}^{-1}$ body weight DDC ex vivo. DDC can enhance macrophage maturation by the induction of 1,25-dihydroxycholecalciferol (vitamin D3) [34]. Vitamin D3 may play an important role in the pathological process of TB by downregulating the levels of MMPs and upregulating the levels of tissue inhibitor of metalloproteinase [79]. Vitamin D3 also mediates the production of cathelicidin, which is a monocyte-macrophage peptide with significant antituberculous activity, in the context of Toll-like receptor 2/1-ligand activation [80]. An in vitro study showed that DDC was highly active against $M$. tuberculosis with a MIC of $8 \mu \mathrm{g} \cdot \mathrm{mL}^{-1}$ [33].

DSF and DDC showed antituberculous activity against more than 40 clinical isolates of M. tuberculosis, including MDR/XDR-TB strains. The MIC90 (MIC required to inhibit growth of $90 \%$ of organisms) of DSF and DDC against clinical isolates were 1.56 and $3.13 \mu \mathrm{g} \cdot \mathrm{mL}^{-1}$, respectively. They also showed bactericidal activity against intracellular $M$. tuberculosis in human monocyte leukaemia cell line (THP-1) at 6-30 $\mu \mathrm{g} \cdot \mathrm{mL}^{-1}$ and $10-30 \mu \mathrm{g} \cdot \mathrm{mL}^{-1}$, respectively [35]. Since no cross resistance of DSF and DDC with other antituberculous drugs was observed, these compounds may be of potential value for future regimens against MDR/XDR-TB [35]. 
In vivo studies

DSF kills $M$. tuberculosis at $80-160 \mathrm{mg} \cdot \mathrm{kg}^{-1}$ in a mouse model with chronic TB [35]. DDC enhances the activity of pyrazinamide and rifampicin twofold when co-administered in mice at $100 \mathrm{mg} \cdot \mathrm{kg}^{-1}$, showing activity against persisters of $M$. tuberculosis [33].

\section{Clinical studies}

DSF is rapidly and completely absorbed following oral administration, and is quickly reduced to DDC. DDC is metabolised to diethylamine, carbon disulfide $\left(\mathrm{CS}_{2}\right)$, DDC methyl ester, DDC glucuronide and DDC sulfate; a small amount of DDC is reoxidised to DSF. DSF, DDC and $\mathrm{CS}_{2}$ are widely distributed throughout the body in lipids of various tissues, and the highest levels of these compounds are found in skeletal muscle [36]. Humans eliminate more than $90 \%$ of orally administered DSF within 3 days by renal clearance as DDC, DDC glucuronide and, to a small extent, as DDC sulfate, and via the breath as $\mathrm{CS}_{2}$ [36]. Few publications showed the activity of DSF or its metabolites against TB. Only one study found that DDC reduces the incidence of infections in HIV co-infected TB patients, probably by stimulating the antimicrobial activity of mononuclear phagocytes [34].

The side-effects of DSF are mainly on the CNS and include psychosis or a confusional state that occurs in the early period of DSF therapy with higher dosages of DSF $\left(500 \mathrm{mg} \cdot \mathrm{day}^{-1}\right)$. Another serious side-effect is peripheral neuropathy. All these side-effects were reversible [81]. Rarely, DSF can cause fatal hepatitis [81].

\section{Co-trimoxazole}

SXT is a synergistic combination of two antimicrobial agents: trimethoprim (TMP) and sulfamethoxazole (SMX). Its mechanism of action is interference with folic acid synthesis in bacteria. SXT is used predominantly to treat urinary tract infections, and for prophylaxis and treatment of Pneumocystis jiroveci pneumonia in HIV patients $[82,83]$. There is ongoing debate about the use of SXT for the treatment of TB. Some studies mention that only SMX was effective against M. tuberculosis, while TMP is not [37, 84].

\section{In vitro studies}

M. tuberculosis strains appeared to be susceptible to SXT in 43 (98\%) out of 44 isolates tested. These isolates were sensitive to TMP/SMX at MIC $\leqslant 1 / 19 \mu \mathrm{g} \cdot \mathrm{mL}^{-1}$ [37]. Another study showed that SMX inhibits $80 \%$ and $99 \%$ growth of all 117 clinical isolates at a MIC of $19 \mathrm{mg} \cdot \mathrm{L}^{-1}$ and $38 \mathrm{mg} \cdot \mathrm{L}^{-1}$, respectively [38]. Drug susceptibility testing in a recent study mentioned that MIC values of SMX for M. tuberculosis ranged between 4.75 and $25 \mu \mathrm{g} \cdot \mathrm{mL}^{-1}$ [55]. In 7H9 broth, M. tuberculosis was susceptible to SMX (MIC90 $\left.8 \mu \mathrm{g} \cdot \mathrm{mL}^{-1}\right)$. In that study, SMX achieved excellent activity against M. tuberculosis [39]. One in vitro study showed that $M$. tuberculosis strain $\mathrm{H} 37 \mathrm{Rv}$ was susceptible to SMX and not to TMP at an MIC of $8.5 \mu \mathrm{g} \cdot \mathrm{mL}^{-1}$. When SXT was added to an isoniazid- or rifampicin-treated $M$. tuberculosis culture isolate, SMX with and without TMP was efficient at killing and preventing its growth, thereby preventing the emergence of drug resistance [40].

In an in vitro study, M. tuberculosis strains were exposed to either TMP/SMX in combination, SMX or TMP alone, or SMX in combination with first-line TB drugs (isoniazid, rifampicin and ethambutol). TMP had a negligible effect on the growth of M. tuberculosis, while SMX inhibited $80 \%$ of the growth at $4.75 \mathrm{mg} \cdot \mathrm{L}^{-1}$. There was no synergistic activity between the TMP and SMX combination, but an additive effect was observed. SMX had a synergistic effect combined with rifampicin, an additive effect combined with ethambutol, and no effect with isoniazid [41].

\section{Clinical studies}

The total absorption of SMX is $85-90 \%$. Concentrations of the non-protein-bound fraction of SMX to TMP in plasma varied between subjects, ranging from 1:5 to 1:40 [42]. The explanation could be that SMX has a smaller volume of distribution (10-20 L) than TMP (69-133 L). In the blood, SMX is bound to plasma proteins to an extent of $58-66 \%$ (34-42\% free) $[42,85]$. SMX is well distributed in most body fluids and also in CSF, so it may be a good candidate for treating TB meningitis [42]. Concentrations of SMX in sputum, middle-ear fluid and paranasal sinus fluid are about $20-27 \%$ of serum, and in bronchial secretion $60-100 \%$ of those in serum [86]. SXT penetrates epithelial lining fluid easily because it is lipophilic and inflammation independent [56,57]. Following distribution of SMX, it is partially acetylated and glucuronide-conjugated in the liver [87]. SMX excretions are 60-65\% acetyl derivative, and 15\% appear as glucuronides. These metabolites constitute $70 \%$ of total SMX in urine and $20-35 \%$ in plasma [42]. The plasma half-life of SMX is $9 \mathrm{~h}[42]$.

Limited data are available on the pharmacokinetic parameters of SXT in MDR-TB patients. Only one study described the pharmacokinetic parameters after receiving $480 \mathrm{mg}$ and $960 \mathrm{mg}$ of SXT. Pharmacokinetic parameters of SMX in TB patients including AUC, clearance and volume of distribution are lower than the 
values observed in patients with other indications. These patients seem to display a consistent pharmacokinetic profile for SMX. SXT was safe and well-tolerated, except for one patient who had gastrointestinal side-effects after receiving $960 \mathrm{mg}$ of SXT [55].

Adherence and tolerance to the drug was good when SXT was used in a daily dose of $960 \mathrm{mg}$ as prophylaxis to reduce the mortality in adults with HIV infection and TB, by preventing opportunistic infections. The rates of occurrence of one or several side-effects that could be due to SXT were similar in the placebo group $(\mathrm{n}=372)$ and in the SXT group $(\mathrm{n}=371)$ [88]. An SXT dose of $960 \mathrm{mg} \cdot \mathrm{day}^{-1}$ added to routine care improved the survival of HIV-positive TB patients dramatically; SXT prophylaxis should therefore be added to the routine care of HIV-positive TB patients [89].

In five clinical studies summarised in one report [83], SXT was safe, feasible and effective in the prophylactic treatment of HIV patients with TB; it has beneficial effects including lower mortality, fewer hospital visits, weight gain and improved CD4 cell counts, and it reduced plasma HIV in $P$. jiroveci pneumonia, malaria and other bacterial (pneumococcal) airway infections. The impact on infections with M. tuberculosis was not considered in these studies.

In general, TMP-SMX is a safe medication and is tolerated well. The most common side-effects include gastrointestinal intolerance, nausea, vomiting, anorexia and diarrhoea [87]. Possible side-effects in the blood are hyperkalaemia, a slight increase in serum creatinine levels (not representing loss of glomerular filtration but rather reversible decrease in tubular excretion of the creatinine molecule) and hypernatraemia. These effects occur especially in patients with renal dysfunction [87, 90, 91]. Haematological abnormalities include leukopenia, agranulocytosis and thrombocytopenia, and haemolytic and aplastic anaemia [92].

\section{Discussion}

The need for new drugs to improve the treatment of patients with MDR-TB has received much attention. These patients are currently treated with a combination of second-line drugs that are more expensive, more toxic and less effective than the drugs used in standard therapy, and which have much longer treatment duration than those for drug-susceptible TB patients. This often results in poor outcomes. Exploring the antimicrobial activity of drugs that are already available on the market would therefore be a tremendous asset. This review analyses the pharmacokinetics and pharmacodynamics, and the in vitro, in vivo and clinical data of these drugs.

Based on the in vitro, in vivo and clinical data of the drugs discussed in this review, thioridazine, doxycycline, metronidazole and SXT were selected as candidate drugs for possible use in MDR-TB. They are effective either against metabolically active, fast-replicating, or metabolically inactive, non-replicating phenotypes of $M$. tuberculosis (dormant state). These drugs also have favourable pharmacokinetics, like CNS and CSF penetration, that may be useful for the treatment of TB meningitis.

However, thioridazine has no activity against rapidly-replicating metabolically active M. tuberculosis, such as inactive cavitary pulmonary $\mathrm{TB}$, because of the poor penetration of this drug into the cavities; it thus lacks efficacy against this stage of TB. The same is true for metronidazole, which is predominantly active under anaerobic conditions, although this drug appears to have activity against dormant $M$. tuberculosis. Compounds of similar structure to metronidazole have been shown to have potent antituberculous activity in vitro and in vivo, like CG17341 and PA 824 [93]. Metronidazole could, thus, be used as a lead compound for the synthesis of new drugs against MDR-TB, especially for dormant organisms. Doxycycline has bacteriostatic but no bactericidal effect against M. tuberculosis, so it is not the first choice to develop further.

According to the pros and cons of the selected drugs mentioned in the Results section, SXT could be the promising drug for treating MDR-TB because of its consistent pharmacokinetic profile in combination with other anti-TB drugs. It is an easy-to-administer, cheap, well-tolerated and safe antimicrobial agent. It has also encouraging activity in vitro. The other compounds discussed here have some limitations that may restrict their use for treatment of MDR-TB. Because of the fatal outcome of a disulfiram-alcohol reaction, this drug should not be administered to TB patients who are alcohol abusers. Tigecycline is administered only parenterally and is not effective against more slow-growing mycobacteria such as M. tuberculosis.

Our review has several strengths. We conducted a very comprehensive literature search by exploring electronic databases to get information about the anti-TB activity of the selected drugs. These drugs are not listed in WHO guidelines but they have in vivo, in vitro or clinical activity against M. tuberculosis, and no previous review mentions their antimicrobial activity in the treatment of MDR-TB. Hence, this review could be an interesting starting point for further research.

This review has some limitations. The review was restricted to only six antimicrobial drugs and does not discuss other new or already-available drugs that have antituberculous activity. Most published articles 
provide insufficient data for the role of these drugs in the treatment of TB. We excluded those articles not available in the English language, even though articles in other languages could have important data. Because limited MDR-TB clinical data for these drugs are available, a lack of dose selection studies was observed. However, we did mention the doses and response to these drugs for TB and XDR-TB patients in clinical studies if they were not available for MDR-TB patients.

There is only limited antimicrobial pharmacokinetic and pharmacodynamic information available for the drugs discussed here. This could be an obstacle for finding suitable doses and predicting their efficacy in the treatment of MDR-TB patients. Antimicrobial pharmacokinetics and pharmacodynamics derived in preclinical pharmacokinetic/pharmacodynamic models like the hollow-fibre infection model and the mouse models, as well as additional clinical trials, do offer the possibility to study toxicity and to determine duration of therapy. These will be important steps in the further development of these drugs for use in MDR-TB.

A clinical prospective study should be conducted to evaluate pharmacokinetic parameters and tolerance in order to find the suitable dose for the treatment of MDR-TB patients in a phase II study. In a phase III study, the clinical efficacy and long-term safety of the drug should be investigated by comparing the sterilising activities of the candidate alone and when substituted for one standard anti-TB drug. These approaches are essential for an efficient clinical development of potential antimicrobial drugs for TB treatment.

\section{References}

1 Keshavjee S, Farmer PE. Tuberculosis, drug resistance, and the history of modern medicine. N Engl J Med 2012; 367: 931-936.

2 Migliori GB, Sotgiu G, D'Ambrosio L, et al. TB and MDR/XDR-TB in European Union and European Economic Area countries: managed or mismanaged? Eur Respir J 2012; 39: 619-625.

3 Skripconoka V, Danilovits M, Pehme L, et al. Delamanid improves outcomes and reduces mortality in multidrugresistant tuberculosis. Eur Respir J 2013; 41: 1393-1400.

4 Leung EC, Leung CC, Kam KM, et al. Transmission of multidrug-resistant and extensively drug-resistant tuberculosis in a metropolitan city. Eur Respir J 2013; 41: 901-908.

5 Orenstein EW, Basu S, Shah NS, et al. Treatment outcomes among patients with multidrug-resistant tuberculosis: systematic review and meta-analysis. Lancet Infect Dis 2009; 9: 153-161.

6 Prabowo SA, Gröschel MI, Schmidt EDL, et al. Targeting multidrug-resistant tuberculosis (MDR-TB) by therapeutic vaccines. Med Microbiol Immunol 2013; 202: 95-104.

World Health Organization. WHO Report on the Tuberculosis Epidemic. Geneva, WHO Press, 1997.

8 World Health Organization. Global Tuberculosis Control Report 2010. (WHO/HTM/TB/2010.7). Geneva, WHO Press, 2010.

9 De Lorenzo S, Alffenaar JW, Sotgiu G, et al. Efficacy and safety of meropenem-clavulanate added to linezolid containing regimens in the treatment of MDR-/XDR-TB. Eur Respir J 2013; 41: 1386-1392.

10 World Health Organization. Global Tuberculosis Report 2012. (WHO/HTM/TB/2012.6). Geneva, WHO Press, 2010.

11 Skrahina A, Hurevich H, Zalutskaya A, et al. Alarming levels of drug-resistant tuberculosis in Belarus: results of a survey in Minsk. Eur Respir J 2012; 39: 1425-1431.

12 Louw GE, Warren RM, Gey van Pittius NC, et al. A balancing act: efflux/influx in mycobacterial drug resistance. Antimicrob Agents Chemother 2009; 53: 3181-3189.

13 Miotto P, Cabibbe AM, Mantegani P, et al. Geno Type MTBDRsl performance on clinical samples with diverse genetic background. Eur Respir J 2012; 40: 690-698.

14 Voskuil MI, Schnappinger D, Visconti KC, et al. Inhibition of respiration by nitric oxide induces a Mycobacterium tuberculosis dormancy program. J Exp Med 2003; 198: 705-713.

15 Tomioka H. Current status of some antituberculosis drugs and the development of new antituberculous agents with special reference to their in vitro and in vivo antimicrobial activities. Curr Pharm Des 2006; 12: 4047-4070.

16 Amaral L, Kristiansen JE, Abebe LS, et al. Inhibition of the respiration of multi-drug resistant clinical isolates of Mycobacterium tuberculosis by thioridazine: potential use for initial therapy of freshly diagnosed tuberculosis. J Antimicrob Chemother 1996; 38: 1049-1053.

17 Martins M, Schelz Z, Martins A, et al. In vitro and ex vivo activity of thioridazine derivatives against Mycobacterium tuberculosis. Int J Antimicrob Agents 2007; 29: 338-340.

18 Martins M, Viveiros M, Kristiansen JE, et al. The curative activity of thioridazine on mice infected with Mycobacterium tuberculosis. In Vivo 2007; 21: 771-775.

19 Dinovo EC, Bost RO, Sunshine I, et al. Distribution of thioridazine and its metabolites in human tissues and fluids obtained postmortem. Clin Chem 1978; 24: 1828-1830.

20 Thanacoody HK. Thioridazine: resurrection as an antimicrobial agent? Br J Clin Pharmacol 2007; 64: 566-574.

21 Wayne LG, Sramek HA. Metronidazole is bactericidal to dormant cells of Mycobacterium tuberculosis. Antimicrob Agents Chemother 1994; 38: 2054-2058.

22 Brooks JV, Furney SK, Orme IM. Metronidazole therapy in mice infected with tuberculosis. Antimicrob Agents Chemother 1999; 43: 1285-1288.

23 Filippini P, Iona E, Piccaro G, et al. Activity of drug combinations against dormant Mycobacterium tuberculosis. Antimicrob Agents Chemother 2010; 54: 2712-2715.

24 Klinkenberg LG, Sutherland LA, Bishai WR, et al. Metronidazole lacks activity against Mycobacterium tuberculosis in an in vivo hypoxic granuloma model of latency. J Infect Dis 2008; 198: 275-283.

25 Hoff DR, Caraway ML, Brooks EJ, et al. Metronidazole lacks antibacterial activity in guinea pigs infected with Mycobacterium tuberculosis. Antimicrob Agents Chemother 2008; 52: 4137-4140.

26 Driver ER, Ryan GJ, Hoff DR, et al. Evaluation of a mouse model of necrotic granuloma formation using C3HeB/ FeJ mice for testing of drugs against Mycobacterium tuberculosis. Antimicrob Agents Chemother 2012; 56: 3181-3195. 
29 2004; 64: 63-88.

30 Conte JE Jr, Golden JA, Kelly MG, et al. Steady-state serum and intrapulmonary pharmacokinetics and pharmacodynamics of tigecycline. Int J Antimicrob Agents 2005; 25: 523-529.

31 Walker NF, Clark SO, Oni T, et al. Doxycycline and HIV infection suppress tuberculosis-induced matrix metalloproteinases. Am J Respir Crit Care Med 2012; 185: 989-997.

32 Balabanova Y, Ruddy M, Hubb J, et al. Multidrug-resistant tuberculosis in Russia: clinical characteristics, analysis of second-line drug resistance and development of standardized therapy. Eur J Clin Microbiol Infect Dis 2005; 24: $136-139$.

33 Byrne ST, Gu P, Zhou J, et al. Pyrrolidine dithiocarbamate and diethyldithiocarbamate are active against growing and nongrowing persister Mycobacterium tuberculosis. Antimicrob Agents Chemother 2007; 51: 4495-4497.

34 Hübner L, Ernst M, von Laer D, et al. Enhancement of monocyte antimycobacterial activity by diethyldithiocarbamate (DTC). Int J Immunopharmacol 1991; 13: 1067-1072.

35 Horita Y, Takii T, Yagi T, et al. Antitubercular activity of disulfiram, an antialcoholism drug, against multidrug- and extensively drug-resistant Mycobacterium tuberculosis isolates. Antimicrob Agents Chemother 2012; 56: 4140-4145.

36 Peachey JE, Brien JF, Roach CA, et al. A comparative review of the pharmacological and toxicological properties of disulfiram and calcium carbimide. J Clin Psychopharmacol 1981; 1: 21-26.

37 Forgacs P, Wengenack NL, Hall L, et al. Tuberculosis and trimethoprim-sulfamethoxazole. Antimicrob Agents Chemother 2009; 53: 4789-4793.

38 Huang TS, Kunin CM, Yan BS, et al. Susceptibility of Mycobacterium tuberculosis to sulfamethoxazole, trimethoprim and their combination over a 12 year period in Taiwan. J Antimicrob Chemother 2012; 67: 633-637.

39 Wallace RJ Jr, Nash DR, Steele LC, et al. Susceptibility testing of slowly growing mycobacteria by a microdilution MIC method with 7H9 broth. J Clin Microbiol 1986; 24: 976-981.

40 Vilchèze C, Jacobs WR Jr. The combination of sulfamethoxazole, trimethoprim, and isoniazid or rifampin is bactericidal and prevents the emergence of drug resistance in Mycobacterium tuberculosis. Antimicrob Agents Chemother 2012; 56: 5142-5148.

41 Macingwana L, Baker B, Ngwane AH, et al. Sulfamethoxazole enhances the antimycobacterial activity of rifampicin. J Antimicrob Chemother 2012; 67: 2908-2911.

42 Reeves DS, Wilkinson PJ. The pharmacokinetics of trimethoprim and trimethoprim/sulphonamide combinations, including penetration into body tissues. Infection 1979; 7: Suppl. 4, S330-S341.

43 Ordway D, Viveiros M, Leandro C, et al. Clinical concentrations of thioridazine kill intracellular multidrugresistant Mycobacterium tuberculosis. Antimicrob Agents Chemother 2003; 47: 917-922.

44 Viveiros M, Amaral L. Enhancement of antibiotic activity against poly-drug resistant Mycobacterium tuberculosis by phenothiazines. Int J Antimicrob Agents 2001; 17: 225-228.

45 Tsuneizumi T, Babb SM, Cohen BM. Drug distribution between blood and brain as a determinant of antipsychotic drug effects. Biol Psychiatry 1992; 32: 817-824.

46 Nyberg G, Axelsson R, Mårtensson E. Binding of thioridazine and thioridazine metabolites to serum proteins in psychiatric patients. Eur J Clin Pharmacol 1978; 14: 341-350.

47 Ginsberg AM. Drugs in development for tuberculosis. Drugs 2010; 70: 2201-2214.

48 Wayne LG, Hayes LG. An in vitro model for sequential study of shiftdown of Mycobacterium tuberculosis through two stages of nonreplicating persistence. Infect Immun 1996; 64: 2062-2069.

49 Jensen JC, Gugler R. Single- and multiple-dose metronidazole kinetics. Clin Pharmacol Ther 1983; 34: $481-487$.

50 Desai CR, Heera S, Patel A, et al. Role of metronidazole in improving response and specific drug sensitivity in advanced pulmonary tuberculosis. J Assoc Physicians India 1989; 37: 694-697.

51 Cina SJ, Russell RA, Conradi SE. Sudden death due to metronidazole/ethanol interaction. Am J Forensic Med Pathol 1996; 17: 343-346.

52 Rubinstein E, Vaughan D. Tigecycline: a novel glycylcycline. Drugs 2005; 65: 1317-1336.

53 Wallace RJ Jr, Brown-Elliott BA, Crist CJ, et al. Comparison of the in vitro activity of the glycylcycline tigecycline (formerly GAR-936) with those of tetracycline, minocycline, and doxycycline against isolates of nontuberculous mycobacteria. Antimicrob Agents Chemother 2002; 46: 3164-3167.

54 Muralidharan G, Micalizzi M, Speth J, et al. Pharmacokinetics of tigecycline after single and multiple doses in healthy subjects. Antimicrob Agents Chemother 2005; 49: 220-229.

55 Alsaad N, van Altena R, Pranger AD, et al. Evaluation of co-trimoxazole in the treatment of multidrug-resistant tuberculosis. Eur Respir J 2013; 42: 504-512.

56 Melmon KL, Carruthers SG, Morrelli HF, et al. Melmon and Morrelli's Clinical Pharmacology: Basic Principles in Therapeutics. 4th Edn. New York, McGraw Hill, 2000.

57 Jarvis WR, ed. Nosocomial Pneumonia. New York, Marcel Dekker, 2000.

58 Abbate E, Vescovo M, Natiello M, et al. Successful alternative treatment of extensively drug-resistant tuberculosis in Argentina with a combination of linezolid, moxifloxacin and thioridazine. J Antimicrob Chemother 2012; 67: 473-477.

59 Sharma S, Singh A. Phenothiazines as anti-tubercular agents: mechanistic insights and clinical implications. Expert Opin Investig Drugs 2011; 20: 1665-1676.

60 Amaral L, Viveiros M, Molnar J. Antimicrobial activity of phenothiazines. In Vivo 2004; 18: 725-731.

61 Amaral L, Viveiros M. Why thioridazine in combination with antibiotics cures extensively drug-resistant Mycobacterium tuberculosis infections. Int J Antimicrob Agents 2012; 39: 376-380.

62 Wójcikowski J, Maurel P, Daniel WA. Characterization of human cytochrome p450 enzymes involved in the metabolism of the piperidine-type phenothiazine neuroleptic thioridazine. Drug Metab Dispos 2006; 34: 471-476.

63 Amaral L, Boeree MJ, Gillespie SH, et al. Thioridazine cures extensively drug-resistant tuberculosis (XDR-TB) and the need for global trials is now!. Int J Antimicrob Agents 2010; 35: 524-526.

64 Mackin P. Cardiac side effects of psychiatric drugs. Hum Psychopharmacol 2008; 23: Suppl. 1, 3-14. 
65 Viveiros M, Martins M, Couto I, et al. The in vitro activity of phenothiazines against Mycobacterium avium: potential of thioridazine for therapy of the co-infected AIDS patient. In Vivo 2005; 19: 733-736.

66 Edwards DI. Mechanism of antimicrobial action of metronidazole. J Antimicrob Chemother 1979; 5: 499-502.

67 Rhoades ER, Frank AA, Orme IM. Progression of chronic pulmonary tuberculosis in mice aerogenically infected with virulent Mycobacterium tuberculosis. Tuber Lung Dis 1997; 78: 57-66.

68 Amon I, Amon K, Hüller H. Pharmacokinetics and therapeutic efficacy of metronidazole at different dosages. Int J Clin Pharmacol Biopharm 1978; 16: 384-386.

69 O'Keefe JP, Troc KA, Thompson KD. Activity of metronidazole and its hydroxy and acid metabolites against clinical isolates of anaerobic bacteria. Antimicrob Agents Chemother 1982; 22: 426-430.

70 Ralph ED, Kirby WM. Bioassay of metronidazole with either anaerobic or aerobic incubation. J Infect Dis 1975; 132: 587-591.

71 Schwartz DE, Jeunet F. Comparative pharmacokinetic studies of ornidazole and metronidazole in man. Chemotherapy 1976; 22: 19-29.

72 Clinicaltrials.gov. Metronidazole for pulmonary tuberculosis (South Korea). ClinicalTrials.gov identifier: NCT00425113. http://clinicaltrials.gov/show/NCT00425113 Date last updated: October 21, 2013.

73 Finegold SM. Metronidazole. Ann Intern Med 1980; 93: 585-587.

74 Sang QX, Jin Y, Newcomer RG, et al. Matrix metalloproteinase inhibitors as prospective agents for the prevention and treatment of cardiovascular and neoplastic diseases. Curr Top Med Chem 2006; 6: 289-316.

75 Vojtova V, Urbanek K. Farmakokinetika tetracyklinu a glycylcyklinu [Pharmacokinetics of tetracyclines and glycylcyclines]. Klin Mikrobiol Infekc Lek 2009; 15: 17-21.

76 Noskin GA. Tigecycline: a new glycylcycline for treatment of serious infections. Clin Infect Dis 2005; 41: Suppl. 5, S303-S314.

77 Smith K, Leyden JJ. Safety of doxycycline and minocycline: a systematic review. Clin Ther 2005; 27: 1329-1342.

78 Maresca A, Carta F, Vullo D, et al. Dithiocarbamates strongly inhibit the $\beta$-class carbonic anhydrases from Mycobacterium tuberculosis. J Enzyme Inhib Med Chem 2013; 28: 407-411.

79 Anand SP, Selvaraj P. Effect of 1, 25 dihydroxyvitamin $\mathrm{D}_{3}$ on matrix metalloproteinases MMP-7, MMP-9 and the inhibitor TIMP-1 in pulmonary tuberculosis. Clin Immunol 2009; 133: 126-131.

80 Liu PT, Stenger S, Li H, et al. Toll-like receptor triggering of a vitamin D-mediated human antimicrobial response. Science 2006; 311: 1770-1773.

81 Chick J. Safety issues concerning the use of disulfiram in treating alcohol dependence. Drug Saf 1999; 20: 427-435.

82 Hawser S, Lociuro S, Islam K. Dihydrofolate reductase inhibitors as antibacterial agents. Biochem Pharmacol 2006; 71: 941-948.

83 Harries AD, Zachariah R, Corbett EL, et al. The HIV-associated tuberculosis epidemic - when will we act? Lancet 2010; 375: 1906-1919.

84 Suling WJ, Reynolds RC, Barrow EW, et al. Susceptibilities of Mycobacterium tuberculosis and Mycobacterium avium complex to lipophilic deazapteridine derivatives, inhibitors of dihydrofolate reductase. J Antimicrob Chemother 1998; 42: 811-815.

85 Patel RB, Welling PG. Clinical pharmacokinetics of co-trimoxazole (trimethoprim-sulphamethoxazole). Clin Pharmacokinet 1980; 5: 405-423.

86 Nightingale CH, Ambrose PG, File TM Jr, eds. Community Acquired Respiratory Infections. New York, Marcel Dekker, Inc., 2003.

87 Smilack JD. Trimethoprim-sulfamethoxazole. Mayo Clin Proc 1999; 74: 730-734.

88 Wiktor SZ, Sassan-Morokro M, Grant AD, et al. Efficacy of trimethoprim-sulphamethoxazole prophylaxis to decrease morbidity and mortality in HIV-1-infected patients with tuberculosis in Abidjan, Côte d'Ivoire: a randomised controlled trial. Lancet 1999; 353: 1469-1475.

89 Mwaungulu FB, Floyd S, Crampin AC, et al. Cotrimoxazole prophylaxis reduces mortality in human immunodeficiency virus-positive tuberculosis patients in Karonga District, Malawi. Bull World Health Organ 2004; 82: 354-363.

90 Masters PA, O’Bryan TA, Zurlo J, et al. Trimethoprim-sulfamethoxazole revisited. Arch Intern Med 2003; 163: 402-410.

91 Mori H, Kuroda Y, Imamura S, et al. Hyponatremia and/or hyperkalemia in patients treated with the standard dose of trimethoprim-sulfamethoxazole. Intern Med 2003; 42: 665-669.

92 Heimpel H, Raghavachar A. Hematological side effects of co-trimoxazole. Infection 1987; 15: Suppl. 5, S248-S253.

93 Barry CE 3rd, Slayden RA, Sampson AE, et al. Use of genomics and combinatorial chemistry in the development of new antimycobacterial drugs. Biochem Pharmacol 2000; 59: 221-231. 